\title{
VERTICAL ACCURACY EVALUATION OF ASTER GDEM2 OVER A MOUNTAINOUS AREA BASED ON UAV PHOTOGRAMMETRY
}

\author{
Yubin Liang ${ }^{a, b, *}$, Yuankun Qu ${ }^{a, b}$, Dongxu Guo ${ }^{a, b}$, Tiejun Cui ${ }^{a, b}$ \\ ${ }^{\text {a }}$ School of Geographic and Environmental Sciences, Tianjin Normal University, Tianjin 300387, China \\ ${ }^{\mathrm{b}}$ Tianjin Engineering Center for Geospatial Information Technology, Tianjin Normal University, Tianjin 300387, China \\ lyb.whu@gmail.com; lily9961@qq.com; 1015881693@qq.com; tiejun_cui@163.com
}

\section{ICWG I/II}

KEY WORDS: Global Digital Elevation Model, ASTER GDEM2, UAV Photogrammetry, Accuracy Evaluation, Geodesy

\begin{abstract}
:
Global digital elevation models (GDEM) provide elementary information on heights of the Earth's surface and objects on the ground. GDEMs have become an important data source for a range of applications. The vertical accuracy of a GDEM is critical for its applications. Nowadays UAVs has been widely used for large-scale surveying and mapping. Compared with traditional surveying techniques, UAV photogrammetry are more convenient and more cost-effective. UAV photogrammetry produces the DEM of the survey area with high accuracy and high spatial resolution. As a result, DEMs resulted from UAV photogrammetry can be used for a more detailed and accurate evaluation of the GDEM product. This study investigates the vertical accuracy (in terms of elevation accuracy and systematic errors) of the ASTER GDEM Version 2 dataset over a complex terrain based on UAV photogrammetry. Experimental results show that the elevation errors of ASTER GDEM2 are in normal distribution and the systematic error is quite small. The accuracy of the ASTER GDEM2 coincides well with that reported by the ASTER validation team. The accuracy in the research area is negatively correlated to both the slope of the terrain and the number of stereo observations. This study also evaluates the vertical accuracy of the up-sampled ASTER GDEM2. Experimental results show that the accuracy of the up-sampled ASTER GDEM2 data in the research area is not significantly reduced by the complexity of the terrain. The fine-grained accuracy evaluation of the ASTER GDEM2 is informative for the GDEM-supported UAV photogrammetric applications.
\end{abstract}

\section{INTRODUCTION}

In the present era of big data, spatial data collection modes are characterized with the progressive course of diversity, efficiency and facilitation, which are producing large sets of data. This further brings the request for advanced data processing with higher level of efficiency, automation and intelligence (Zhang and Tao, 2017). In the past decade, significant advances in global elevation modelling have been made with the release of ASTER (Advanced Spaceborne Thermal Emission and Reflection Radiometer) GDEM and SRTM (Shuttle Radar Topography Mission) GDEM. Global digital elevation models (GDEM) provide elementary information on heights of the Earth's surface and ground objects, which have become an important data source for a range of applications such as surveying and mapping, gravity-field modelling, hydrological studies, flood simulation, emergency response and many more (Teo et al., 2010). The vertical accuracy of a GDEM is critical for its applications. In the field of UAV photogrammetry, flight planners usually consider the average elevation of the whole surveying area. However, the relative flight heights of UAVs are usually lower than those of manned aircrafts. The complexity of the terrain may result in serious occlusions and under-overlap due to low flight heights. Footprints and Voronoi diagrams have been used to generate image pairs for image matching. Footprint-based method generated the footprints images and then determined the image pairs by footprint intersection (Rupnik et al., 2014). The terrain is usually assumed planar for this kind of methods. If the terrain is complex, the footprints will not lie on a plane, which makes it difficult to determine the intersections. DEMs can also be used for direct georeferencing. DEM-based direct georeferencing usually starts from an average elevation and iterates to converge. However, the iterative method may stop at a local optimal with bad initialization due to complex terrain and inaccurate Positioning and Orientation data. All of these applications including flight planning, plan evaluation, image matching, direct georeferencing and ortho-mosaicing can be improved by using simulation methods with GDEM data (Liang et al., 2017). However, the accuracies of the simulations are largely dependent on the vertical accuracy of the GDEM data used.

Since 2000 the Japanese ASTER instrument, payload on NASA's Terra satellite, acquires stereo image data with its two nadir- and backward-viewing telescopes, which are sensitive in the near infrared spectral band. The joint Japanese-US ASTER GDEM version 2 was released in October 2011 by NASA and the Ministry of Economy Trade and Industry (METI) of Japan. ASTER GDEM2 covers most of the earth surface and is freely available all over the world. The ASTER GDEM data is acquired through stereo observations and requires a large number of accurate ground control points to ensure its worldwide accuracy. With high spatial resolution $(\sim 30 \mathrm{~m})$, the ASTER GDEM2 has been widely used. The basic features of ASTER GDEM2 are listed in (Tachikawa et al., 2011a).

Generally, high-precision ground control points are used to evaluate the accuracy of GDEM products. There have existed a

\footnotetext{
* Corresponding author
} 
number of researches that evaluated the accuracies of the GDEMs. Hirt et al. (2010) compared ASTER GDEM (version 1), the SRTM DEM (version 4.1) and the GEODATA DEM-9S (version 3), and evaluated them using 6392 levelling and 911 GPS/levelling ground control points.

The second version of ASTER GDEM is reported to have improved significantly with respect to its predecessor in terms of vertical height bias, striping error and voids that have been filled to some extent (Krieger et al., 2010; Carabajal, 2011; Gesh et al., 2011; Tachikawa et al., 2011a). In a summarising study by the joint Japan-US ASTER Science Team comprising a total of four independent validation studies, the vertical accuracy of ASTER GDEM2 is estimated to be around $17 \mathrm{~m}$ at a confidence interval of 95\% (Tachikawa et al., 2011b). Rexer and Hirt (2014) evaluated the accuracy of the ASTER GDEM2 and SRTM v2.1/v4.1 over Australia using ground truth data from the Australian National Gravity Database. The research found that the elevation accuracy of these datasets were at the level of meters. However, measuring ground control points is costly and the ground sample interval is relatively low. Although ASTER GDEM2 has an overall accuracy of around $17 \mathrm{~m}$ at the $95 \%$ confidence level, the accuracy of ASTER GDEM2 in a certain area is affected by the observation conditions, the complexity and land cover of the terrain, and the number and distribution of control points.

Nowadays UAVs has been widely used for large-scale surveying and mapping (Haala et al., 2011; Remondino et al., 2011; Colomina and Molina, 2014). Compared with traditional surveying techniques, UAV photogrammetry are more convenient and more cost-effective. UAV photogrammetry produces the DEM of the survey area with high accuracy and high spatial resolution. As a result, DEMs resulted from UAV photogrammetry can be used for a more detailed and accurate evaluation of the GDEM product.

This study investigates the vertical accuracy (in terms of elevation accuracy and systematic errors) of the ASTER GDEM Version 2 dataset over a complex and vegetation-covered terrain. The accuracy of the ASTER GDEM2 data is evaluated with a high resolution DEM produced by UAV photogrammetry. Section 2 details the methodology of the study. Experimental results are discussed in Section 3 and conclusion is made in Section 4.

\section{METHODOLOGY}

The production of the DEM by photogrammetry mainly consists of image matching, relative orientation, absolute orientation, dense matching and raster DEM gridding. In the image matching procedure, the image pairs are generated by using a simulation method. A set of points were sampled from the ASTER GDEM2 data and then transformed to the East-NorthUp (ENU) coordinate system. The positions of the images are also transformed from the geodetic coordinate system to ENU. The orientations of the images are used to construct the rotation matrix R defined in the collinearity equation (equation (1)). The collinearity equation is a mathematical model which projects a three dimensional point $\mathrm{P}$ onto the image plane. Figure 1 illustrates the geometric configuration of perspective projection defined by the collinearity equation.

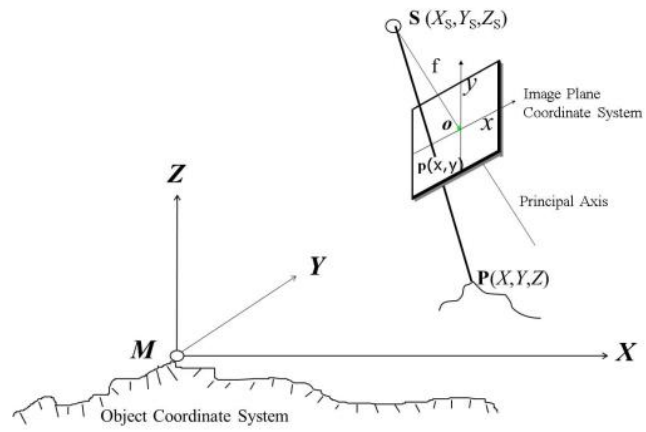

Figure 1. The geometric configuration of perspective projection defined by the collinearity equation

$$
\left.\begin{array}{l}
x=-f \frac{a_{1}\left(X-X_{S}\right)+b_{1}\left(Y-Y_{S}\right)+c_{1}\left(Z-Z_{S}\right)}{a_{3}\left(X-X_{S}\right)+b_{3}\left(Y-Y_{S}\right)+c_{3}\left(Z-Z_{S}\right)} \\
y=-f \frac{a_{2}\left(X-X_{S}\right)+b_{2}\left(Y-Y_{S}\right)+c_{2}\left(Z-Z_{S}\right)}{a_{3}\left(X-X_{S}\right)+b_{3}\left(Y-Y_{S}\right)+c_{3}\left(Z-Z_{S}\right)}
\end{array}\right\}
$$

where $(\mathrm{X}, \mathrm{Y}, \mathrm{Z})$ is the position of a three dimensional point $\mathrm{P}$ under the object coordinate system $\mathrm{M}-\mathrm{XYZ},(\mathrm{x}, \mathrm{y})$ is the position of $\mathrm{p}$ under the image plane coordinate system o-xy, $\left(\mathrm{X}_{\mathrm{S}}\right.$, $\mathrm{Y}_{\mathrm{S}}, \mathrm{Z}_{\mathrm{S}}$ ) is the position of the center of projection $\mathrm{S}$ under $\mathrm{M}$ $\mathrm{XYZ}, \mathrm{f}$ is the focal length, and the nine parameters (a1-c3) are the elements of the rotation matrix $\mathrm{R}$ defined as

$$
R=\left[\begin{array}{lll}
a_{1} & a_{2} & a_{3} \\
b_{1} & b_{2} & b_{3} \\
c_{1} & c_{2} & c_{3}
\end{array}\right]
$$

where

$$
\left.\begin{array}{l}
a_{1}=\cos \varphi \cos \kappa-\sin \varphi \sin \omega \sin \kappa \\
a_{2}=-\cos \varphi \sin \kappa-\sin \varphi \sin \omega \cos \kappa \\
a_{3}=-\sin \varphi \cos \omega \\
b_{1}=\cos \omega \sin \kappa \\
b_{2}=\cos \omega \cos \kappa \\
b_{3}=-\sin \omega \\
c_{1}=\sin \varphi \cos \kappa+\cos \varphi \sin \omega \sin \kappa \\
c_{2}=-\sin \varphi \sin \kappa+\cos \varphi \sin \omega \cos \kappa \\
c_{3}=\cos \varphi \cos \omega
\end{array}\right\}
$$

where $\varphi$ is the primary rotation about the Y-axis, $\omega$ is the secondary rotation about the $\mathrm{X}$-axis, and $\kappa$ is the tertiary rotation about the Z-axis.

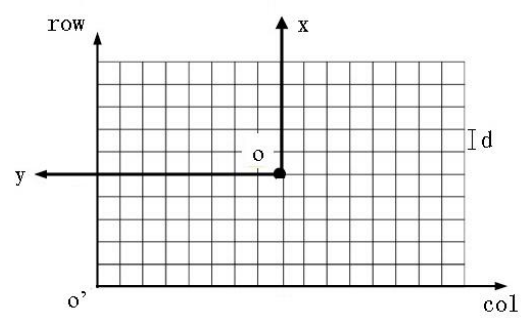

Figure 2. The image plane coordinate system and pixel coordinate system

When all of the parameters in equation (1) are known, the projection $\mathrm{p}$ of a ground point $\mathrm{P}$ can be determined. Given the relationship between the image plane coordinate system o-xy and the pixel coordinate system o'-colrow shown in Figure 2, the position of $p$ under o'-colrow can be determined by equation (4). 


$$
\left.\begin{array}{l}
c o l=\operatorname{col} l_{p p}-\frac{y}{d} \\
r o w=\operatorname{row}_{p p}+\frac{x}{d}
\end{array}\right\}
$$

where $\left(\mathrm{col}_{\mathrm{pp}}\right.$, row $\left._{\mathrm{pp}}\right)$ is the position of the principal point under $\mathrm{o}^{\prime}$-colrow, $(\mathrm{x}, \mathrm{y})$ is the position of $\mathrm{p}$ under $\mathrm{o}-\mathrm{xy}$, and $\mathrm{d}$ is the pixel size.

The image observations of the ground sample points are simulated by using the collinearity equation. The images with projections of the same ground sample points are considered as overlapped. The image pairs generated with the POS and DEM data are accurate and false image pairs are effectively filtered.

The tie points generated by the image matching procedure are then used for relative orientation. After the relative orientation, the positions and orientations of images under a local coordinate system are determined by an incremental bundle block adjustment procedure.

The result of the relative orientation and the coordinates of the GCPs are then used for absolute orientation. After the absolute orientation, the positions and orientations of images under ENU coordinate system are determined by a bundle block adjustment.

The dense matching is then carried out to generate the dense cloud of the research area. The Triangulated Irregular Network (TIN) is generated from the resulting dense point cloud. On the basis of the TIN, a raster DEM is interpolated and output as a GeoTIFF file.

To evaluate the vertical accuracy of the ASTER GDEM2 data, the DEM produced by UAV photogrammetry is down-sampled to the resolution of ASTER GDEM2 data. Then pixel-wise errors are derived from the ASTER DEM and the UAV DEM. The RMSE is then derived from the errors. The slope map of the research area is derived from the UAV DEM. The correlation matrix between the errors of ASTER DEM and the slopes is calculated and analyzed. The QA file with the ASTER GDEM2 data indicates the number of ASTER stereo scene pairs ("stacking number") used to determine elevation at given pixel. In this study, the correlation matrix between the errors of ASTER DEM and the stacking numbers is also calculated and analyzed.

The ASTER GDEM2 can be used for the direct georeferencing and ortho-mosaicing. In these applications, the ASTER DEM data is usually up-sampled. This study also evaluates the vertical accuracy of the up-sampled ASTER DEM. The ASTER DEM is up-sampled to the resolution of UAV DEM. Then pixel-wise errors are derived by subtracting the UAV DEM from the ASTER DEM. The RMSE is then derived from the errors and compared with the aforementioned down-sampled result.

\section{RESULTS AND DISCUSSION}

The rectangle-like research area is $6 \mathrm{~km}$ from east to west and 7 $\mathrm{km}$ from south to north (Figure 3). The elevation of the area is in the range of $[200,1050]$ meters. Most of the research area is covered by shrubs and trees.

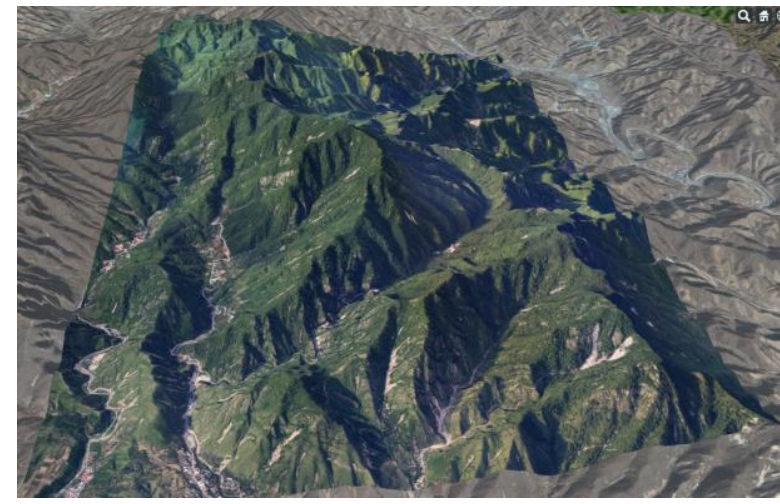

Figure 3. The research area shown in 3D

The ASTER GDEM2 data of the research area was downloaded from the web as a GeoTIFF file. ASTER refers to WGS84, with the heights transformed via EGM96 to a physical height. The ASTER GDEM2 data of the research area is shown in Figure 4.

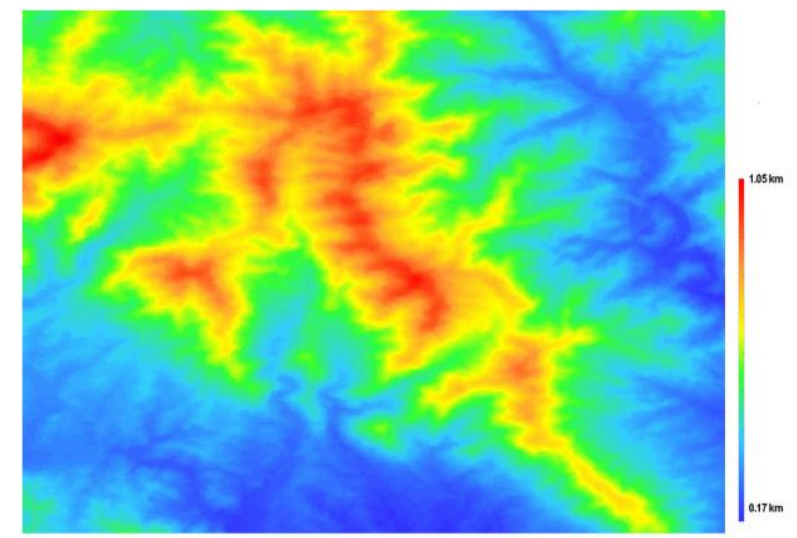

Figure 4. The ASTER GDEM2 data of the research area

The aerial images were taken by a Nikon D810 camera with a $35 \mathrm{~mm}$ fixed focal lens mounted on a fixed-wing UAV in autumn 2016. The flight height was about 1500 meters above the sea level. During the flight, the positions and orientations of the images were also recorded. The forward/side overlapping of the flight is about $80 \% .1198$ valid images were acquired in total. The image resolution is 7360 by 4912 . The spatial resolution of the images is about $0.2 \mathrm{~m}$. Figure 5 shows one of the images captured.

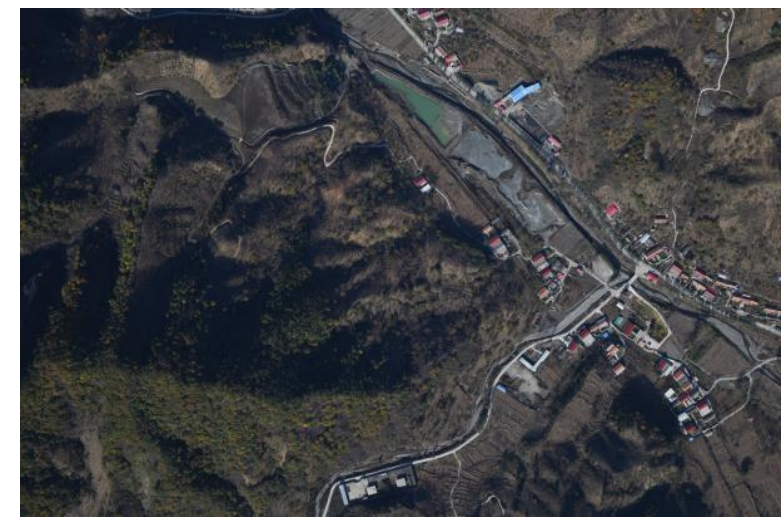

Figure 5. One of the UAV images 
Figure 6 shows the camera locations and number of image observations of the research area. It can be seen that most of the research area can be observed by more than 9 images.
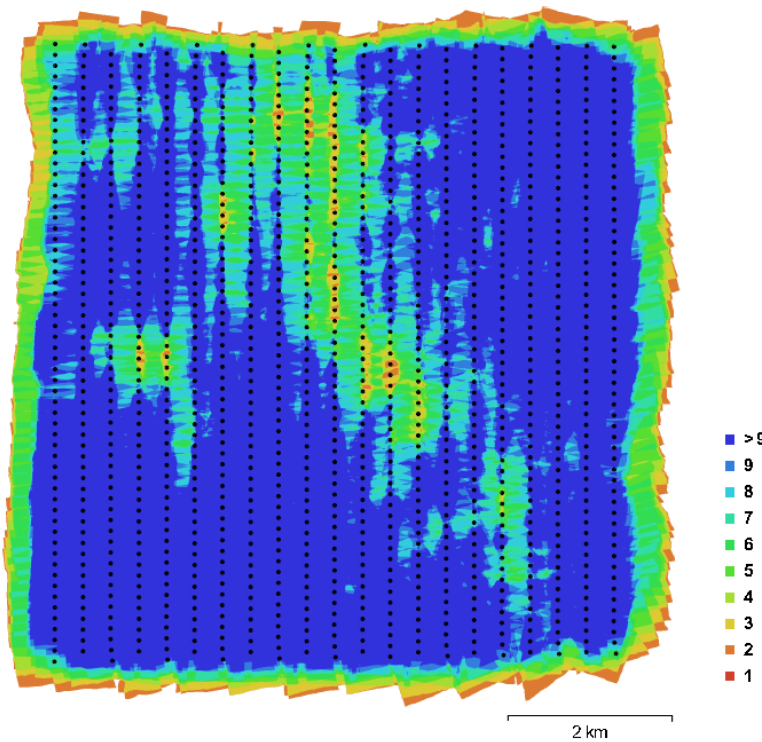

Figure 6. Camera locations and number of image observations of the research area

Seven ground control points (K1-K7) were measured under WGS84 coordinate system by using the static networked GPS and the accuracy of the ground control points were at the level of decimetres. Figure 7 shows the positions of the GCPs.

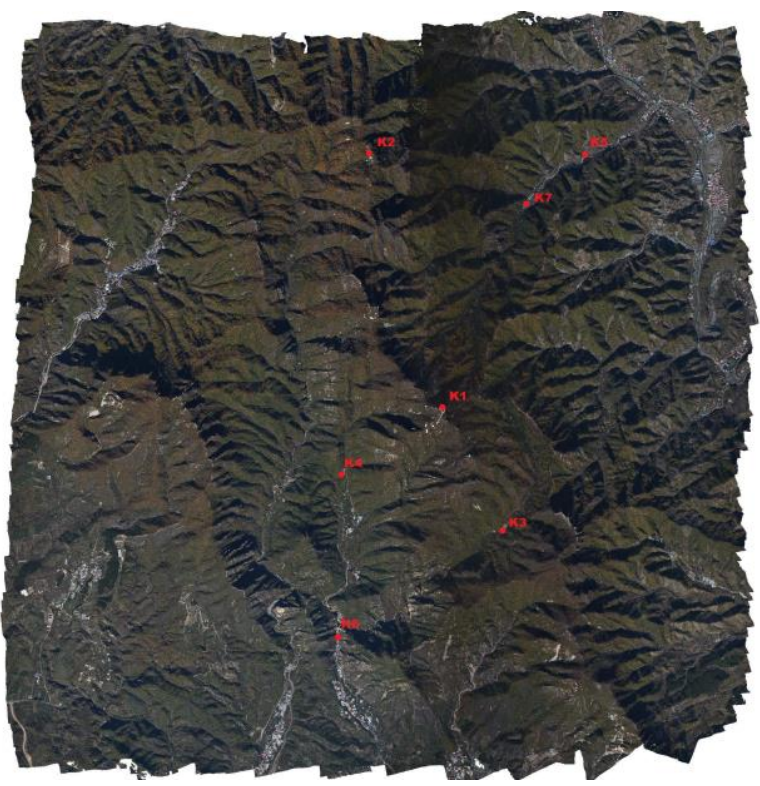

Figure 7. Positions of the GCPs

The accuracy of the aerial triangulation at control points after the bundle block adjustment is given by Table 1 . The maximum absolute $\mathrm{Z}$ error is $0.703 \mathrm{~m}$ and the RMSE along $\mathrm{Z}$ axis is $0.278 \mathrm{~m}$. It can be seen that the vertical accuracy of the aerial triangulation is at the level of decimetres, which is accurate enough to evaluate the ASTER GDEM2 data.

Table 1. The accuracy of the aerial triangulation on the GCPs \begin{tabular}{|l|l|l|l|l|l|}
\hline Label & $\mathrm{X}$ error (m) & Y error (m) & Z error (m) & Total (m) & Image (pix) \\
\hline
\end{tabular}

\begin{tabular}{|c|c|c|c|c|c|}
\hline K1 & 0.001 & -0.003 & 0.023 & 0.023 & $0.119(2)$ \\
\hline K2 & 0.005 & -0.006 & 0.097 & 0.097 & $0.211(2)$ \\
\hline K3 & 0.092 & -0.019 & 0.049 & 0.106 & $0.210(7)$ \\
\hline K4 & -0.047 & -0.047 & -0.068 & 0.095 & $0.230(9)$ \\
\hline K5 & 0.020 & -0.092 & 0.166 & 0.190 & $0.428(9)$ \\
\hline K6 & -0.036 & 0.064 & 0.018 & 0.076 & $0.379(9)$ \\
\hline K7 & 0.066 & 0.178 & -0.703 & 0.729 & $0.359(4)$ \\
\hline Total & 0.049 & 0.082 & 0.278 & 0.294 & 0.322 \\
\hline
\end{tabular}

Figure 8 shows the raster DEM with $0.3 \mathrm{~m}$ spatial resolution produced by UAV photogrammetry.

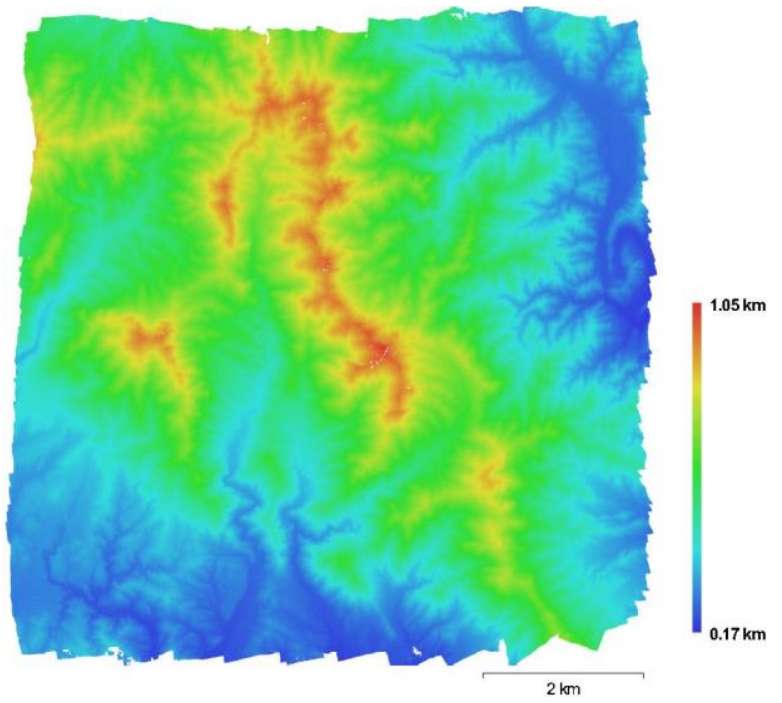

Figure 8 . The $0.3 \mathrm{~m}$ DEM produced by UAV photogrammetry

To evaluate the vertical accuracy of the ASTER GDEM2 data, the DEM produced by UAV photogrammetry is down-sampled to $30 \mathrm{~m}$ by nearest neighbor interpolation.

The elevation values at the edges of the DEM are not reliable due to few image observations (Figure 6). Therefore, the UAV DEM is masked to clip the edges. After the masking process, pixel-wise errors are derived by subtracting the UAV DEM from the ASTER DEM. Figure 9 shows the spatial distribution of the errors.

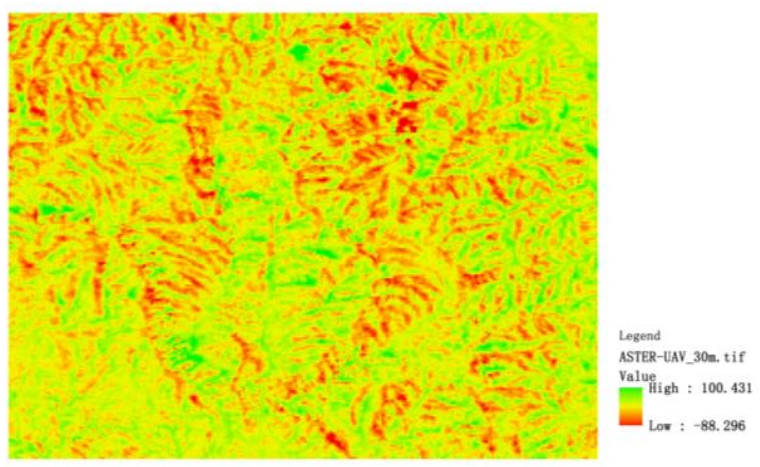

Figure 9. The spatial distribution of the errors derived from the ASTER DEM and the UAV DEM (30m resolution)

The maximum and the minimum error are $100.43 \mathrm{~m}$ and $88.30 \mathrm{~m}$ respectively. The histogram of the errors is shown in Figure 10. Statistical test shows that the errors come from a 
normal distribution with a mean of $0.06 \mathrm{~m}$ and a standard deviation of $15.04 \mathrm{~m}$, which indicates that the systematic error is quite small. The RMSE is $15.04 \mathrm{~m}$, which coincides well with the $15.85 \mathrm{~m}$ accuracy reported by (Tachikawa et al., 2011a).

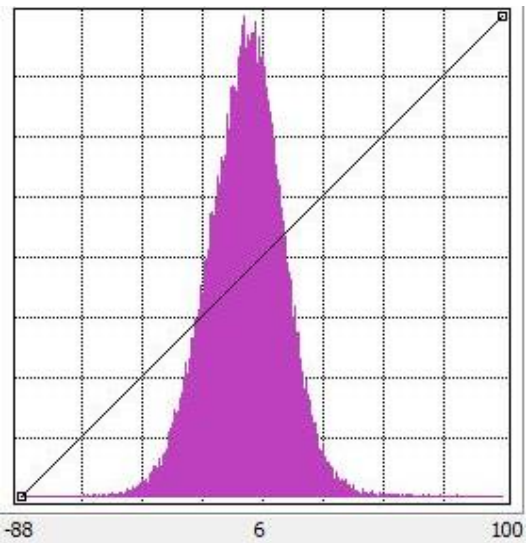

Figure 10. The histogram of the errors ( $30 \mathrm{~m}$ resolution)

Figure 11 shows the slope map of the research area derived from the UAV DEM (30m resolution). It can be seen that the slopes of most areas are greater than 30 degrees.
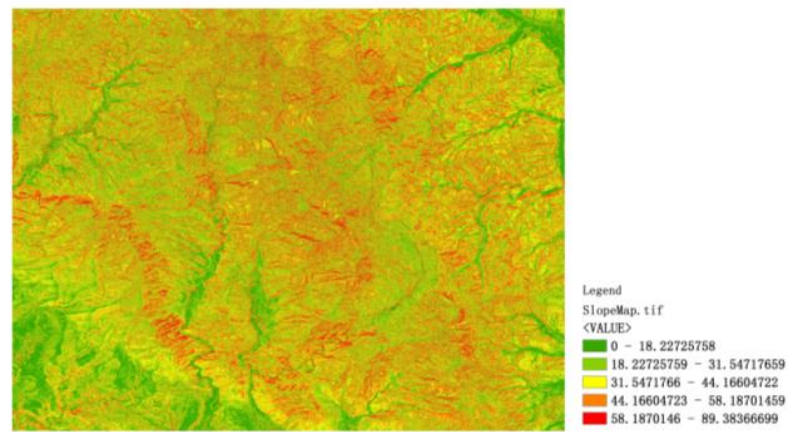

Figure 11. The slope map of the research area (Unit: degrees)

Table 2 shows the statistics of the errors of ASTER GDEM and the slopes. The maximum and the minimum slope are 0 and 89.3837 degrees respectively. The mean and the standard deviation of the slopes are 35.7365 and 16.6769 degrees. The correlation coefficient between the errors of ASTER DEM and the slopes is -0.034 , which indicates that the accuracy of the ASTER GDEM2 data in the research area is negatively correlated to the slope of the terrain. The errors tend to be large in the flat areas and small in the rough areas. It can be seen from Figure 3 and Figure 11 that most of the flat areas are in the valleys. The large errors at these areas may be caused by worse observation condition.

Table 2. Statistics of the slopes and the errors

\begin{tabular}{|c|c|c|c|c|}
\hline Layer & MIN & MAX & MEAN & STD \\
\hline $\begin{array}{c}\text { Slopes } \\
\text { degrees) }\end{array}$ & 0.0000 & 89.3837 & 35.7365 & 16.6769 \\
\hline Errors (m) & -88.30 & 100.43 & 0.06 & 15.04 \\
\hline \multicolumn{4}{|c|}{ CORRELATION MATRIX } \\
\hline Layer & \multicolumn{2}{|c|}{ Slopes (degrees) } & Errors (m) \\
\hline $\begin{array}{c}\text { Slopes } \\
\text { degrees) }\end{array}$ & \multicolumn{2}{|c|}{1.000} & -0.034 \\
\hline Errors (m) & -0.034 & 1.000 \\
\hline
\end{tabular}

The stacking map of the research area is derived from the QA file with the ASTER GDEM2 data (30m resolution). The stacking map is shown in Figure 12. It can be seen that the stacking number of most areas is larger than 4 .

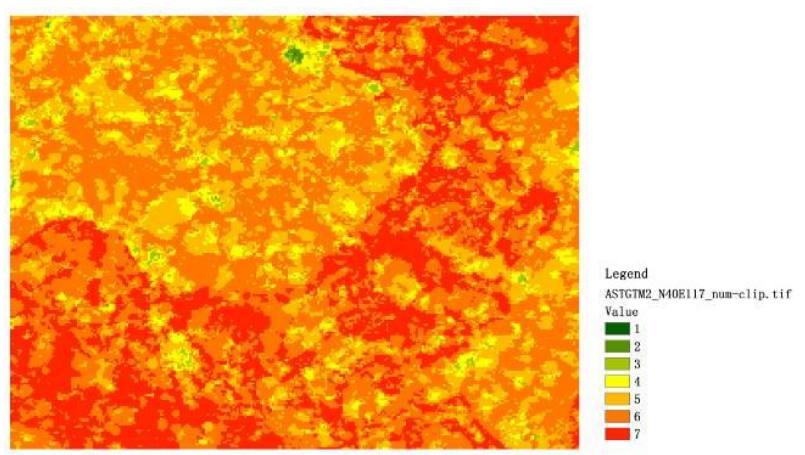

Figure 12. The stacking map of the ASTER GDEM2 data

Table 3 shows the statistics of the errors of ASTER GDEM and the stacking numbers. The maximum and the minimum stacking number are 7 and 1 respectively. The mean and the standard deviation of the stacking numbers are 5.8178 and 0.8690 . The correlation coefficient between the errors of ASTER DEM and the stacking numbers is -0.009 , which indicates that the accuracy of ASTER GDEM2 is negatively correlated to the number of stereo observations. The errors tend to be large in the areas that less observed and small otherwise.

Table 3. Statistics of the stacking numbers and the errors

\begin{tabular}{|c|c|c|c|c|}
\hline Layer & MIN & MAX & MEAN & STD \\
\hline $\begin{array}{c}\text { Stacking } \\
\text { Numbers }\end{array}$ & 1 & 7 & 5.8178 & 0.8690 \\
\hline Errors (m) & -88.30 & 100.43 & 0.06 & 15.04 \\
\hline & \multicolumn{3}{|c|}{ CORRELATION MATRIX } \\
\hline Layer & \multicolumn{2}{|c|}{ Stacking Numbers } & Errors (m) \\
\hline $\begin{array}{c}\text { Stacking } \\
\text { Numbers }\end{array}$ & \multicolumn{2}{|c|}{1.000} & -0.009 \\
\hline Errors (m) & \multicolumn{2}{|c|}{-0.009} & 1.000 \\
\hline
\end{tabular}

To evaluate the vertical accuracy of the up-sampled ASTER DEM, the ASTER DEM data is up-sampled to $0.3 \mathrm{~m}$ by nearest neighbor interpolation. The pixel-wise errors are derived by subtracting the UAV DEM from the ASTER DEM. Figure 13 shows the spatial distribution of the errors.

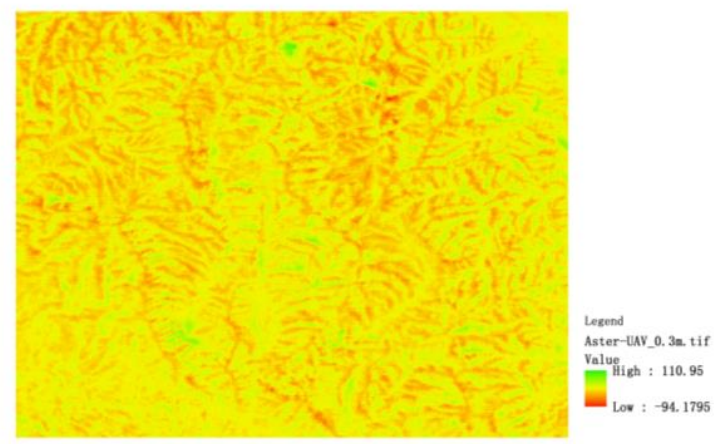

Figure 13. The spatial distribution of the errors derived from the ASTER DEM and the UAV DEM (0.3m resolution) 
The maximum and the minimum error are $110.95 \mathrm{~m}$ and $94.18 \mathrm{~m}$ respectively. The histogram of the errors is shown in Figure 14. Statistical test shows that the errors come from a normal distribution with a mean of $0.68 \mathrm{~m}$ and a standard deviation of $15.22 \mathrm{~m}$. Although the RMSE $(15.22 \mathrm{~m})$ is larger than that of the down-sampled result $(15.04 \mathrm{~m})$, it seems that the accuracy of the up-sampled ASTER GDEM2 data in the research area is not significantly reduced by the complexity of the terrain.

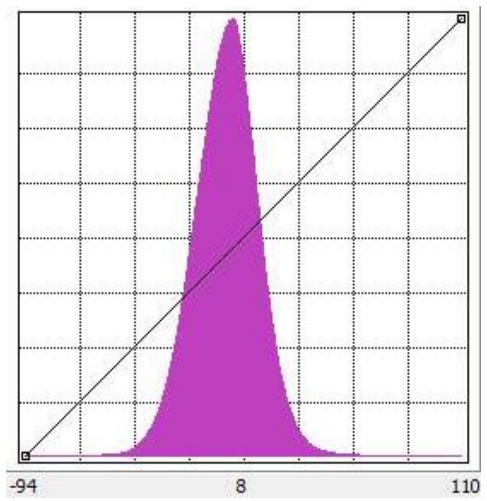

Figure 14 . The histogram of the errors $(0.3 \mathrm{~m}$ resolution)

\section{CONCLUSIONS AND FUTURE WORK}

Global digital elevation models have become an important data source for a range of applications. This study investigates the vertical accuracy of the ASTER GDEM2 over a mountainous area based on UAV photogrammetry. Experimental results show that the elevation errors of ASTER GDEM2 are in normal distribution and the systematic error is quite small. The accuracy of the ASTER GDEM2 coincides well with that reported by the ASTER validation team. It is worth mentioning that the accuracy in the research area is negatively correlated to both the slope of the terrain and the number of stereo observations. This study also evaluates the vertical accuracy of the up-sampled ASTER DEM. Although the accuracy is a little lower than that of the down-sampled result, it seems that the accuracy of the up-sampled ASTER GDEM2 data in the research area is not significantly reduced by the complexity of the terrain.

This study provides fine-grained accuracy evaluation of the ASTER GDEM2 and is informative for the GDEM-supported UAV photogrammetric applications. The future work will focus on accuracy analysis of POS-supported bundle block adjustment, direct georeferencing and ortho-mosaicing.

\section{ACKNOWLEDGMENT}

This work is supported by the National Natural Science Foundation of China under Grant 41471314 and Tianjin Science Project under Grant 15ZCZDSF00390.

\section{REFERENCES}

Carabajal, C., 2011. ASTER global DEM version 2.0 evaluation using ICESat geodetic ground control. Report to the ASTER GDEM Version 2.

Colomina, I., Molina, P., 2014. Unmanned aerial systems for photogrammetry and remote sensing: A review. ISPRS Journal of photogrammetry and remote sensing 92, 79-97.
Gesch, D., Oimoen, M., Zhang, Z., Danielson, J., Meyer, D., 2011. Validation of the ASTER Global Digital Elevation Model (GDEM) Version 2 over the Conterminous United States. Report to the ASTER GDEM Version 2.

Haala, N., Cramer, M., Weimer, F., Trittler, M., 2011. Performance test on UAV-based photogrammetric data collection. Proceedings of the International Archives of the Photogrammetry, Remote Sensing and Spatial Information Sciences 38, 7-12.

Hirt, C., Filmer, M.S., Featherstone, W.E., 2010. Comparison and validation of the recent freely available ASTER-GDEM ver1, SRTM ver4.1 and GEODATA DEM-9S ver3 digital elevation models over Australia. Australian Journal of Earth Sciences 57, 337-347.

Krieger, T., Curtis, W., Haase, J., 2011. Global Validation of the ASTER Global Digital Elevation Model (GDEM) Version 2. Report to the ASTER GDEM Version 2.

Liang, Y., Qu, Y., Cui, T., 2017. A THREE-DIMENSIONAL SIMULATION AND VISUALIZATION SYSTEM FOR UAV PHOTOGRAMMETRY. Int. Arch. Photogramm. Remote Sens. Spatial Inf. Sci. XLII-2/W6, 217-222.

Remondino, F., Barazzetti, L., Nex, F., Scaioni, M., Sarazzi, D., 2011. UAV photogrammetry for mapping and $3 \mathrm{~d}$ modelingcurrent status and future perspectives. International Archives of the Photogrammetry, Remote Sensing and Spatial Information Sciences 38, C22.

Rexer, M., Hirt, C., 2014. Comparison of free high resolution digital elevation data sets (ASTER GDEM2, SRTM v2. 1/v4. 1) and validation against accurate heights from the Australian National Gravity Database. Australian Journal of Earth Sciences 61, 213-226.

Rupnik, E., Nex, F., Remondino, F., 2014. Oblique multicamera systems-orientation and dense matching issues. The International Archives of Photogrammetry, Remote Sensing and Spatial Information Sciences 40, 107.

Tachikawa, T., Hato, M., Kaku, M., Iwasaki, A., 2011a. Characteristics of ASTER GDEM version 2, 2011 IEEE International Geoscience and Remote Sensing Symposium, pp. $3657-3660$

Tachikawa, T., Kaku, M., Iwasaki, A., Gesch, D.B., Oimoen, M.J., Zhang, Z., Danielson, J.J., Krieger, T., Curtis, B., Haase, J., 2011b. ASTER global digital elevation model version 2summary of validation results. NASA.

Teo, T.A., Chen, L.C., Liu, C.L., Tung, Y.C., Wu, W.Y., 2010. DEM-Aided Block Adjustment for Satellite Images With Weak Convergence Geometry. IEEE Transactions on Geoscience and Remote Sensing 48, 1907-1918.

Zhang, Z., Tao, P., 2017. An Overview on "Cloud Control" Photogrammetry in Big Data Era. Acta Geodaetica et Cartographica Sinica 46, 1238-1248. 